\title{
Spatiotemporal optical solitons in nonlinear dissipative media: From stationary light bullets to pulsating complexes
}

\author{
Nail Akhmediev \\ Optical Sciences Group, Research School of Physical Sciences and Engineering, \\ The Australian National University, Canberra ACT 0200, Australia \\ J. M. Soto-Crespo \\ Instituto de Óptica, C.S.I.C., Serrano 121, 28006 Madrid, Spain \\ Philippe Grelu \\ Institut Carnot de Bourgogne, Unité Mixte de Recherche 5209 du C.N.R.S, Université de Bourgogne, \\ BP 47870, 21078 Dijon Cedex, France
}

(Received 19 February 2007; accepted 15 May 2007; published online 28 September 2007)

\begin{abstract}
Nonlinear dissipative systems display the full $(3+1) \mathrm{D}$ spatiotemporal dynamics of stable optical solitons. We review recent results that were obtained within the complex cubic-quintic GinzburgLandau equation model. Numerical simulations reveal the existence of stationary bell-shaped $(3+1) \mathrm{D}$ solitons for both anomalous and normal chromatic dispersion regimes, as well as the formation of double soliton complexes. We provide additional insight concerning the possible dynamics of these soliton complexes, consider collision cases between two solitons, and discuss the ways nonstationary evolution can lead to optical pattern formation. () 2007 American Institute of Physics. [DOI: 10.1063/1.2746830]
\end{abstract}

The subject of dissipative solitons has emerged recently, driving an impressive number of studies in many areas of nonlinear science. Previous studies of purely temporal dissipative solitons have been highly useful, for both fundamental science and the development of high bit-rate optical telecommunications and passively mode-locked lasers. Now, it is the time for generalizations to $(3+1)$-D domain. An appropriate term to describe the new structures is "dissipative optical bullets." Spatiotemporal optical dissipative solitons are localized formations in systems with gain, loss, spectral filtering, dispersion, diffraction, and nonlinearity. The combined interplay between these physical effects creates an optical field confined in both temporal and transverse coordinates. The confinement occurs mainly due to the nonlinear gain balanced with a nonlinear loss in the system. The balance between nonlinearity and dispersion/diffraction, which was the main reason for the existence of conservative solitons, can be weakened or even lifted. Consequently, dissipative optical bullets can exist in media with either anomalous or normal dispersion. Optical bullets have extended regions of stability, which allow experimental observation and potential use of these objects. We present here a review of the latest numerical results concerning dissipative spatiotemporal optical solitons and their complexes. The interaction between stable dissipative light bullets reveals the importance of the phase difference between them. Controlling this parameter can lead to fusion, erasure of one of the solitons or to the formation of soliton complexes, or bound states. Collisions of solitons with nonzero velocities can also create these bound states. The bound states in turn can exhibit a wide range of dynamics, such as rotation and vibration, making them akin to atomic molecules. Among other interesting dy- namical aspects of dissipative optical bullets presented in this work is a transverse pattern formation out of the light bullets that lack stability.

\section{INTRODUCTION}

Dissipative systems are common in nature. Strictly speaking, any other physical model is just an idealization. The so-called conservative or Hamiltonian systems provide convenient models for basic mathematical analysis of simple motion, but they fail to describe real dynamics in longer time scales. Observing nature, we can realize that "particles" are always submerged into dissipative media, which feed their continuous motion. Abstracting ourselves from idealizations we can also notice that this motion is mostly collective and externally driven. A common delusion about dissipative systems is that they can only have losses and thus only decaying solutions. The name "dissipative system" was coined by Nikolis and Prigogine ${ }^{1}$ for systems considered in nonequilibrium thermodynamics. These systems are not isolated, but are kept in contact with an external source that provides energy for the smaller subsystem. Thus, dissipation is essential for the transfer of pumped energy to a "cooler." Hence the notion "dissipative system" is more complicated. It assumes that there is also an energy supply part, rather than just losses. Localized structures in these systems, i.e., "dissipative solitons," have gained their own right to become an established scientific keyword. ${ }^{2}$

The term "optical bullet" was proposed by Silberberg ${ }^{3}$ to depict a complete spatiotemporal soliton, for which confinement in the three spatial dimensions and localization in the temporal domain are achieved by the balance between a focusing nonlinearity and the spreading due to chromatic dis- 
persion and angular diffraction. This occurs while propagation takes place in the medium. For conservative Kerr media, the central issue is the problem of collapse. A cubic-quintic medium that features strong saturation of the Kerr effect could avoid collapse and provide confinement in several dimensions. ${ }^{4-7}$ However, such a medium is difficult to find in practice. Photorefractive materials possess strongly saturating nonlinearity but their slow response restrict their use to the formation of spatial solitons. ${ }^{8}$ Quadratic materials are possible alternative fast media, in which spatial solitons consist of mutually trapped fundamental and second-harmonic waves. ${ }^{9-14}$ Experimentally, $(2+1) \mathrm{D}$ solitons in this arrangement have been observed, ${ }^{10,11}$ but $(3+1) \mathrm{D}$ spatiotemporal solitons, or light bullets, have not yet been reported experimentally. A great difficulty with quadratic nonlinearity is to find suitable material and pulse conditions to phase match fundamental and second-harmonic waves. Recent attempts include the consideration of nonlinear conical waves. ${ }^{15,16}$

Dissipative nonlinear systems suggest an interesting alternative. The additional balance between gain and loss in dissipative systems provides the shape rigidity of the bullet. In the $(3+1) \mathrm{D}$ spatiotemporal case, they can be called optical dissipative bullets. Rosanov has shown that dissipative optical systems can admit solitons in one, two, and three dimensions. ${ }^{17}$ These formations are stable on propagation, provided the system parameters are chosen in specific regions. In addition to stationary solitons, there are pulsating ones, which also exist in separate regions of the parameter space. For one-dimensional (1D) and two-dimensional (2D) solitons, the regions of their existence have been studied extensively, whereas the three-dimensional (3D) case is still mainly beyond our knowledge. Dissipative light bullets can be considered as extensions of "cavity solitons." These cavity solitons are inherently spatial dissipative solitons that were predicted and observed in various resonator configurations, such as in semiconductor optical cavities. ${ }^{18,19}$ Arising from the realistic point of view of a laser cavity, the terminology "cavity light bullets" has been introduced. ${ }^{20,21}$

Dissipative solitons can be considered as elementary building blocks of more complicated structures and patterns in dissipative systems. Indeed, the pattern formation phenomenon is much the same as crystallization in solid-state physics when atoms are combined into periodic structures. Similarly, dissipative solitons can be combined into "molecules" and patterns. Thus, the knowledge of their properties is crucial for understanding the behavior of the system itself. Stable solitons usually have a fixed shape, and the way they are combined into pairs and higher-order composite structures is one of the major questions in these studies.

With rare exceptions, ${ }^{2}$ dissipative systems are nonintegrable. Exact analytical soliton solutions do exist in the case of the one-dimensional cubic-quintic complex GinzburgLandau equation (CGLE), although all of them have proved to be unstable. ${ }^{22}$ Stable solutions of the $(1+1)-\mathrm{D}$ CGLE were found only numerically, and it is expected that, in the two- and three-dimensional cases the only hope to find localized structures is by using numerical simulations. Recently, we have numerically demonstrated the existence of stable light bullets using the three-dimensional CGLE model in both regimes of chromatic dispersion. ${ }^{23,24}$ We also studied temporal elongation processes of optical bullets in the normal dispersion regime (transformation into "rockets"). In the present paper, we concentrate on the conditions of coexistence of an optical dissipative bullet and a double bullet complex, as well as on the process of the spontaneous transformation of a single bullet into a double bullet complex. A double bullet complex is a localized structure that can be also stationary, pulsating, and rotating. Each behavior is primarily defined by the choice of the parameters of the system and to a lesser extent by the choice of the initial condition. The stability region of double bullet complexes can overlap with the stability region for single bullets. This causes them to exist simultaneously and creates the possibility of mutual transformations.

The energy of a double bullet complex can be constant or oscillate with single or double periods. When the total energy of a double bullet complex is constant, it is higher than the sum of the energies of two separate bullets, thus indicating a positive binding energy of the complex. The existence of a binding energy in a double bullet complex calls for an analogy with the case of a diatomic molecule. Although there are similarities, there are marked differences too: A stable diatomic molecule of matter can be considered as an isolated system in which the total energy is conserved, and the binding energy should be negative. A double dissipative bullet complex is an open system, which requires a continuous supply of energy, and there are no conserved quantities for this system. However, the possible existence of long-term stability of these complexes makes the comparison with molecules sustainable, and the analogy can be even quite useful when studying the possible internal pulsations of complexes, as we shall see in Secs. IV and V.

Recently, the "optical soliton molecule" terminology appeared explicitly in the titles of published literature and was employed to depict stable aggregates in 1D or 3D conservative nonlinear systems. ${ }^{25,26}$ We should emphasize that in the case of conservative nonlinear systems, there is only marginal stability, so that Hamiltonian "optical molecules" are not stationary and turn to be decaying in the long term. In contrast, our study indicates that the long-term stability of a dissipative optical molecule relies, when it exists, on the existence of an attractor for the evolution of the dynamical system. Following Silberberg, ${ }^{3}$ we use the term "light bullet" for the $(3+1) \mathrm{D}$ spatiotemporal optical soliton. In view of the above discussion, in our case the term "dissipative light bullet" is more appropriate.

\section{THE GINZBURG-LANDAU PROPAGATION MODEL OF WAVE PROPAGATION FOR $(3+1)$-D DISSIPATIVE MEDIA}

Our studies of dissipative light bullets are based on an extended CGLE model. This model describes a wide range of physical phenomena in dissipative systems. It includes cubic and quintic nonlinearities of dispersive and dissipative types, and we have added transverse operators to take into account spatial diffraction in the paraxial wave approximation. The normalized propagation equation reads 


$$
\begin{aligned}
i \psi_{z} & +\frac{D}{2} \psi_{t t}+\frac{1}{2} \psi_{x x}+\frac{1}{2} \psi_{y y}+|\psi|^{2} \psi+\nu|\psi|^{4} \psi \\
& =i \delta \psi+i \epsilon|\psi|^{2} \psi+i \beta \psi_{t t}+i \mu|\psi|^{4} \psi .
\end{aligned}
$$

The optical envelope $\psi$ is a complex function of four real variables, $\psi=\psi(x, y, t, z)$, where $t$ is the retarded time in the frame moving with the pulse, $z$ is the propagation distance, and $x$ and $y$ are the two transverse coordinates. Equation (1) is written in normalized form. The left-hand-side contains the conservative terms, viz., $D=+1(-1)$, which is for the anomalous (normal) dispersion propagation regime and $\nu$, which is the saturation coefficient of the Kerr nonlinearity. In the following, the dispersion can have either sign, and the saturation of the Kerr nonlinearity is kept relatively small. The right-hand-side includes all dissipative terms: $\delta, \epsilon, \beta$, and $\mu$ are the coefficients for linear loss (if negative), nonlinear gain (if positive), spectral filtering, and saturation of the nonlinear gain (if negative), respectively.

This distributed equation could be applied to the modeling of a wide-aperture laser cavity in the short pulse regime of operation. The model includes the effects of twodimensional transverse diffraction of the beam, longitudinal dispersion of the pulse, and its evolution along the cavity. Dissipative terms describe the gain and loss of the pulse in the cavity. Higher-order dissipative terms are responsible for the nonlinear transmission characteristics of the cavity, which allows, for example, passive mode locking. This equation is a natural extension of the one-dimensional complex cubic-quintic Ginzburg-Landau equation.

We have solved Eq. (1) using a split-step Fourier method. Thus, the second-order derivative terms in $x, y$, and $t$ are solved in Fourier space. Consequently, we apply periodic boundary conditions in $x, y$, and $t$. All other linear and nonlinear terms in the equation are solved in real space using a fourth-order Runge-Kutta method. Most of the simulations presented in the paper were done using a numerical grid of 256 points in each of the three dimensions $x, y$, and $t$. We used various values of step sizes along the spatial and temporal domains to check that the results do not depend on the mesh intervals, thus avoiding any numerical artifacts. A typical numerical run presented in this work takes from several hours to several days on a standard modern PC.

In the $(1+1)$-D case, the cubic-quintic CGLE admits soliton solutions. Moreover, several solutions can exist for the same set of parameters. ${ }^{27}$ Not all of them are necessarily stable. The stability is controlled by the parameters of the equation and by the choice of the soliton branch. In this paper we deal with $(3+1)$-D solitons or optical bullets. We showed in Refs. 23 and 24 that, in the case of anomalous dispersion as well as for normal dispersion, Eq. (1) admits 3D dissipative solitons, i.e., optical bullets. This equation also has stable pulsating solutions in both regimes of dispersion. Pulsating solutions turned out to be double bullet complexes. The first step is to find a set of parameters where stationary or pulsating solitons exist. In general, we fix five of the parameters, namely $D, \mu, \nu, \delta, \beta$, and change $\epsilon$ when looking for stable localized solutions. The initial conditions must be localized when we are looking for localized structures. Their exact shape is relevant but plays a secondary role if only one type of optical bullet exists for a given set of parameters. The shape becomes highly important when several stable solutions coexist. Once a certain kind of localized solution is found for a given set of equation parameters, it can serve as the initial condition for finding solutions at other nearby values of the parameters. By moving slowly in the parameter space, we are able to determine the regions of soliton existence in a relatively easy way.

The natural control parameter of the solution as it evolves is the total energy $Q$, given by the three-dimensional integral of $|\psi|^{2}$ over $x, y$, and $t$,

$$
Q(z)=\int_{-\infty}^{\infty}|\psi(x, y, t, z)|^{2} d x d y d t
$$

For a dissipative system, the energy is not conserved but evolves in accordance with the so-called balance equation. ${ }^{28}$ If the solution stays localized, the energy evolves but remains finite. Furthermore, when a stationary solution is reached, the energy $Q$ converges to a constant value. When the optical field spreads out, the energy tends to infinity. Another possibility is that the solution dissipates and then the energy goes to zero. However, if the optical bullet is a pulsating one, the energy $Q$ is an oscillating function of $z$. We observed all these scenarios in numerical simulations.

\section{STABLE STATIONARY "OPTICAL BULLETS" IN ALL DISPERSION REGIMES}

One decade ago, studies of the (1+1)-D CGLE proved that true bright temporal solitons in a normally dispersive medium can exist, provided that nonlinear dissipation plays a key role. ${ }^{29}$ Exact analytical soliton solutions were found, although all of them proved to be unstable. Numerical simulations allowed one to find regions in the parameter space, where stable temporal solitons exist. These stable solitons exhibit pulse shapes that vary from "bell-shaped" to "flat-top" temporal profiles, depending on the equation parameters. ${ }^{29}$

The results presented in this section can be considered as the extension to the $(3+1)$-D case of these "bright normal dissipative temporal solitons." In particular, we show that, for the medium modeled by Eq. (1), bell-shaped profiles are found in both temporal and spatial transversal coordinates, in contrast to the conical shape of $X$ waves generated in quadratic normally dispersive media. ${ }^{24}$ Furthermore, we also show parameters for which the light bullet is not stable in the temporal domain, where its shape evolves towards a rocket. ${ }^{24}$ Additionally, we present some features of the interaction between stable dissipative optical bullets.

\section{A. Stable and unstable stationary bullets}

The numerical search of a stationary light bullet can be a lengthy procedure, since one has to get a suitable set of parameters for Eq. (1), in the sense that the signs and relative values should be meaningful, and the whole set should provide stationary light bullets for a significantly large range of initial conditions.

Once such set is found, one should pay attention to the type of initial condition. If a cylindrically symmetric initial 

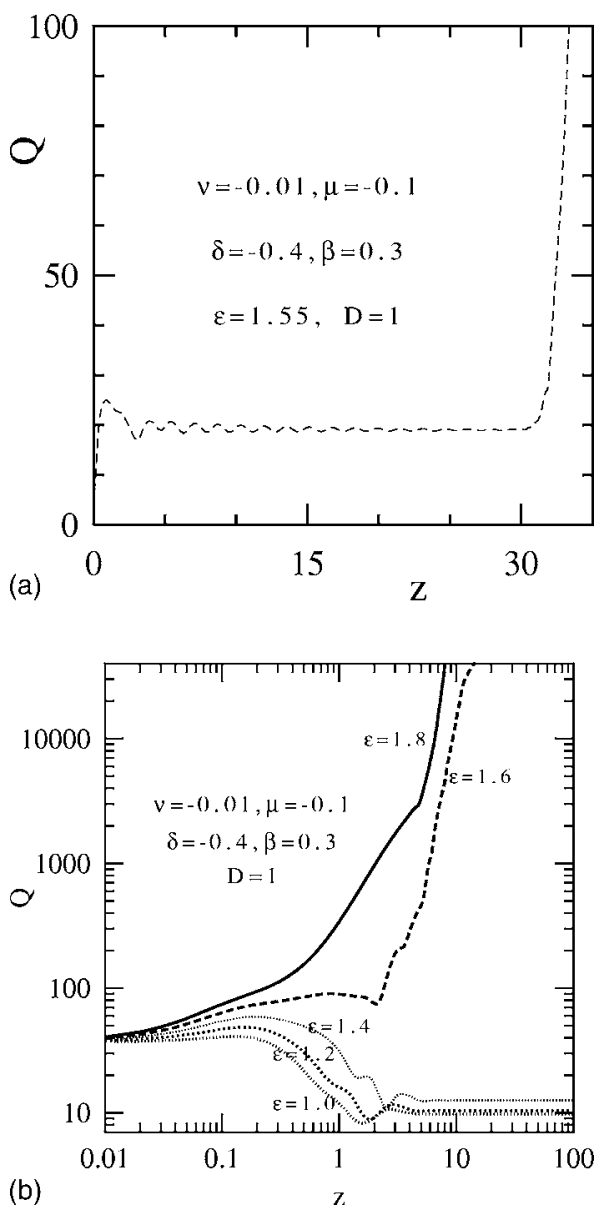

FIG. 1. Evolution of the field energy with distance of propagation $z$. Parameters of simulation are given in each plot. (a) The stationary localized solution reached in this case proves to be unstable at $z=30$. (b) Influence of the nonlinear gain parameter on the excitation of stable soliton solution.

condition is chosen, it can excite a cylindrically symmetric stationary light bullet that nevertheless could be unstable with respect to asymmetric perturbations. This case is illustrated in Fig. 1(a), which plots the evolution of the total energy $Q$ with respect to the propagated distance $z$. The initial condition possesses cylindrical symmetry,

$$
\psi(x, y, t, 0)=1.8 e^{-t^{2}-x^{2}-y^{2}} .
$$

The initial condition Eq. (3) is not a stationary solution, but a stationary localized solution exists in its neighborhood. Therefore, the field evolves until it converges to an optical bullet. Figure 1(a) shows that after some initial variation of $Q(z)$, it converges oscillatorily to a fixed value that corresponds to the energy of the stationary solution. The stationary solution has a cylindrical symmetry that is conserved until the numerical noise breaks it at approximately $z=30$. Up to this value, the solution propagates as an optical bullet without any visible changes. This numerical example shows that unstable bullets can be excited under proper symmetric initial conditions.

If such an unstable light bullet is found, a working strategy to find stable bullets generally consists of decreasing the nonlinear gain parameter, or increasing the saturation of the nonlinear gain. This strategy is illustrated in Fig. 1(b), where the nonlinear gain $\varepsilon$ is decreased from 1.8 to 1.0 , with the rest of the parameters being fixed. The initial condition this time is chosen as a transversally asymmetric one, in order to prevent the excitation of stationary but unstable bullets as described above,

$$
\psi(x, y, t, 0)=4 \exp \left[-\left(\frac{t}{1.2}\right)^{2}-x^{2}-\left(\frac{y}{1.2}\right)^{2}\right] .
$$

The three lower values of $\varepsilon(1.0,1.2$, and 1.4) provide convergence of this initial condition to stable optical bullets. The energy becomes a constant after $z$ reaches the value of approximately 10 in the simulations. The transverse and temporal profiles of the field envelope of the light bullet, which is obtained for $\varepsilon=1.4$, is displayed in Fig. 2(a). The solutions of the optical bullets found in the interval $\varepsilon=1.0-1.4$ have similar bell-shaped profiles. Thus, we can see evidence of excitation of stable optical bullets. This happens for a wide range of the nonlinear gain parameter.

\section{B. Bell-shaped bullets in all dispersion regimes}

Optical bullets exist in finite regions in the parameter space, but it would be extremely difficult to map in extenso these regions in the six-dimensional (6D) parameter space in which we operate. Generally, we restrict ourselves to varying two parameters while fixing the other four. We have chosen the dispersion $D$ and the cubic gain $\varepsilon$ as the variable parameters. With the spectral filtering parameter $\beta$ positive, it is possible to renormalize the time variable in Eq. (1) in such a way that the relevant parameter is simply $D / \beta$. This effectively reduces the dimension of the parameter space to 5 . We view the dispersion coefficient $D$ and the nonlinear gain coefficient $\varepsilon$ as the main parameters that can be easily changed in most experiments such as with passively mode-locked lasers. We have determined the regions of existence of stable bullets following the same technique that we used for dissipative solitons in the $(1+1)$-dimensional case. ${ }^{29} \mathrm{~A}$ twodimensional slice of this region is shown in Fig. 3. The region is relatively wide in both the $\varepsilon$ and $D / \beta$ directions. Inside this region, light bullet solutions are characterized by plain, bell-shaped profiles. Examples of temporal and spatial transverse profiles for zero dispersion are given in Fig. 2(b), while the profiles for normal dispersion are given in Fig. 2(c). A general observed trend is that when moving from anomalous to normal dispersion regime, the shape of the temporal profile departs from the shape of the transverse profile, and bears a more or less pronounced flat-top profile. This is clearly seen in Fig. 2, whereas the transverse profile is the same whatever the dispersion sign is.

In the anomalous dispersion regime, a relatively small influence of the spectral filtering term results in a close-tospherical symmetry in Eq. (1), which in turn results in similar temporal and transverse profiles. Naturally, when the dispersion moves in the normal regime, this "almost symmetry" between temporal and transverse variables is completely broken, so the profiles adopt significantly different shapes. As we shall see in Sec. IV B, this situation can go up to a point 

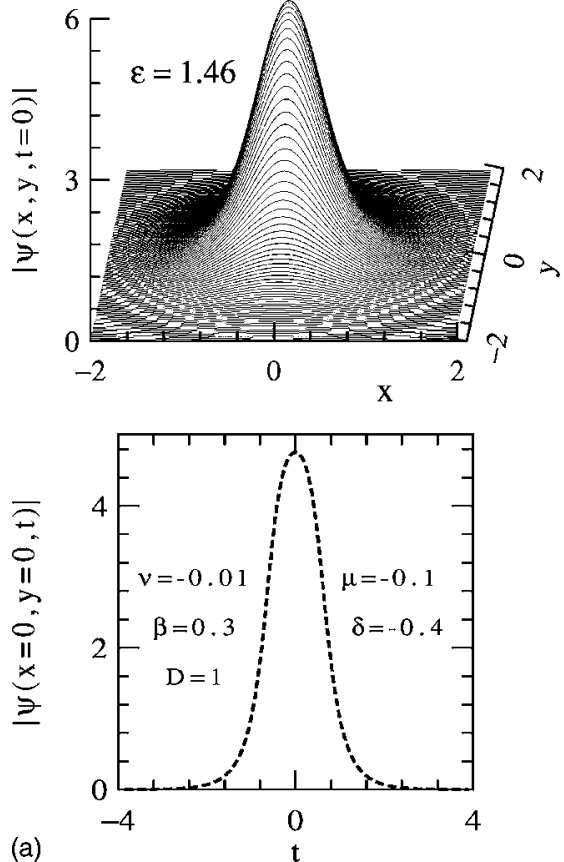
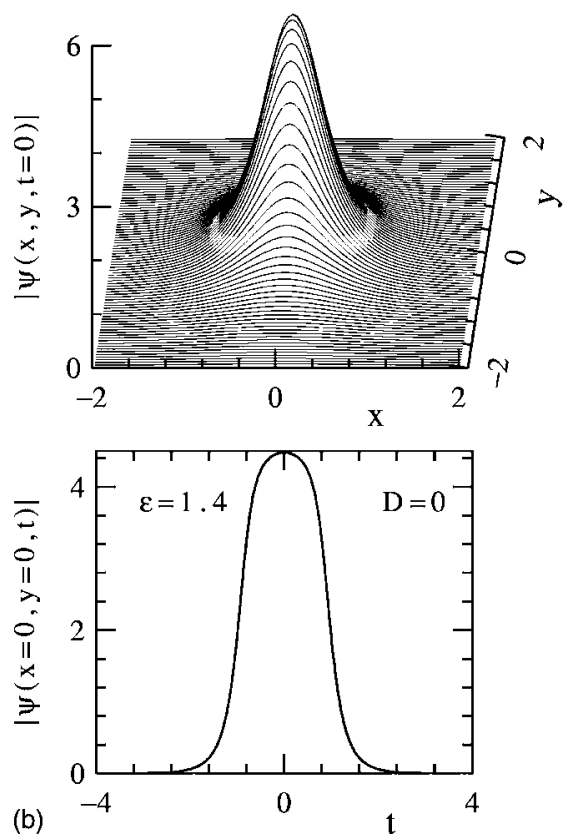
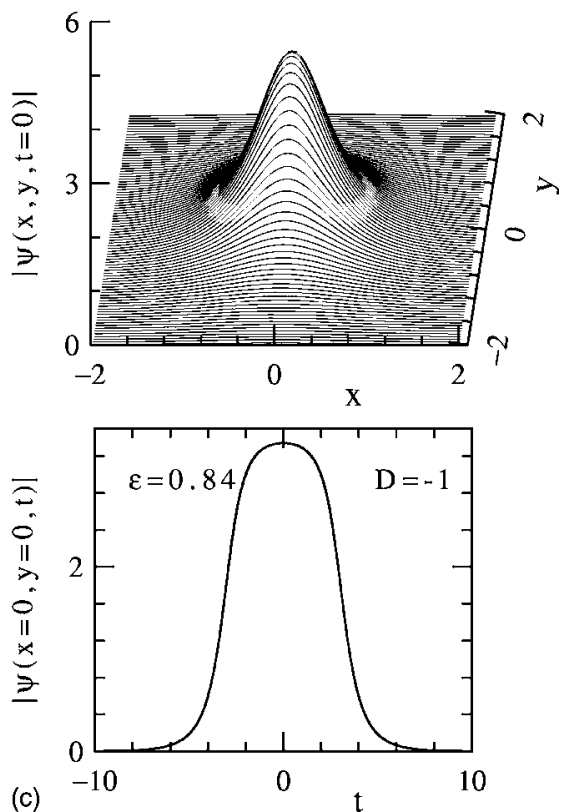

FIG. 2. Bell-shaped dissipative light bullets in propagation regime of (a) anomalous dispersion, (b) zero dispersion, and (c) normal dispersion.

where the temporal profile becomes largely nonstationary, whereas the transverse profile behaves almost stationarily, for specific sets of parameters.

The conclusion to this section is that the region of stable existence of light bullets extends to both positive and negative values of the dispersion parameter. Thus, we could expect the observation of light bullets in the normal and anomalous dispersion regimes of a laser cavity setup. This observation confirms the fact that the main balance necessary for the formation of a localized solution in dissipative systems is between gain and loss. The balance between nonlinearity and dispersion that played a major role in conservative systems is partially lifted in dissipative media with anomalous dispersion, and completely lifted in dissipative media with normal dispersion. However, Fig. 3 indicates that negative $D$ values result in a smaller region of soliton existence

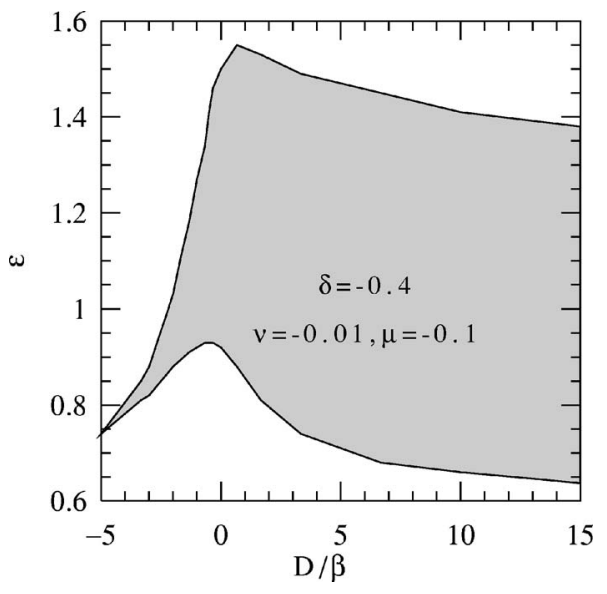

FIG. 3. Existence domain of the stable stationary light bullet, with respect to the nonlinear gain parameter and the ration between the dispersion coefficient and the spectral filtering coefficient. due to the lifting of the balance between dispersion and nonlinearity. The latter makes conditions for soliton creation more difficult.

\section{Basin of attraction}

Figure 4(a) shows an example of the evolution of the energy $Q$ along $z$ towards a stationary solution, when we
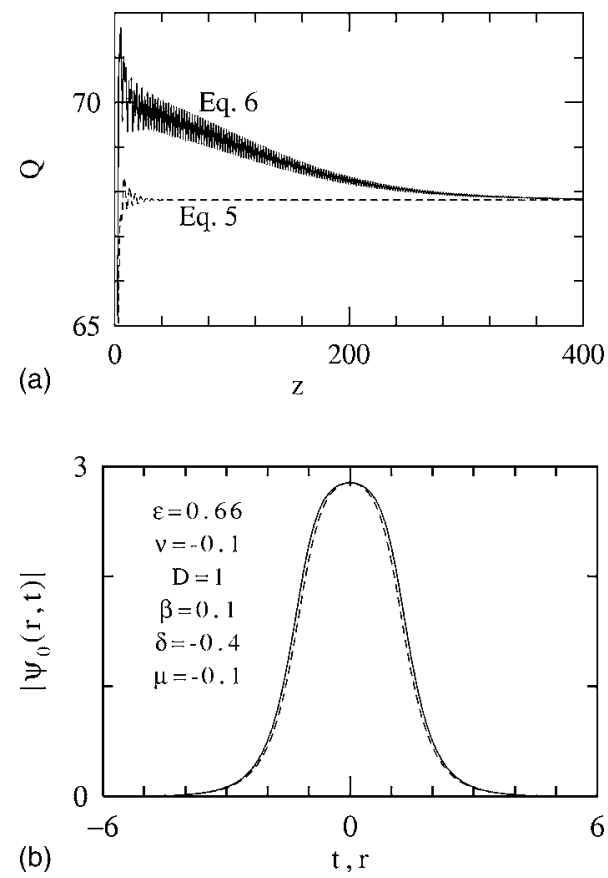

FIG. 4. (a) Convergence of two initial conditions with spherical (lower curve) and elliptical (upper curve) transverse profiles towards the same stationary dissipative light bullet. Spatial and temporal profiles of the bullet are shown in (b) by the dashed and solid lines, respectively. The profiles are almost identical in this example. 
start the simulation with two different localized initial conditions, such as with a Gaussian profile in the three dimensions. Specifically, the two initial conditions that we have chosen are

$$
\psi(x, y, t, 0)=4 \exp \left[-t^{2}-\left(\frac{x}{1.2}\right)^{2}-\left(\frac{y}{1.2}\right)^{2}\right]
$$

and

$$
\psi(x, y, t, 0)=4 \exp \left[-t^{2}-\left(\frac{x}{1.1}\right)^{2}-\left(\frac{y}{1.5}\right)^{2}\right]
$$

The initial condition, Eq. (5), has an energy lower than the energy of the stationary bullet for the chosen set of parameters. However, this initial condition belongs to the basin of attraction of the stationary bullet. Figure 4(a) shows that the energy $Q$ initially increases, and finally converges to a constant value, thus confirming the fact that the localized solution itself tends to converge to a stationary profile. As expected, the stationary solution is a radially symmetric object in the $(x, y)$ plane. The radial (dashed line) and temporal (solid line) profiles of this stationary solution are shown in Fig. 4(b). These curves represent $\left|\psi_{o}(r, t)\right|$ versus $r=\sqrt{x^{2}+y^{2}}$ at $t=0$ and $\left|\psi_{o}(r, t)\right|$ at $r=0$, respectively. The subscript 0 indicates the asymptotic profile when $z \rightarrow \infty$, i.e., when the stationary solution has been reached. The parameters of the simulation are written inside Fig. 4(b). We can see that, in this case, the temporal profile of the bullet is very similar to, though slightly broader than, that of the radial profile. This is a good illustration of the close-to-spherical symmetry consideration discussed in the previous paragraph. Although the initial condition, Eq. (5), is transversally symmetric, the extent of the length of the propagated distance indicates that the stationary asymptotic solution is most likely stable, in contrast to the situation presented in Fig. 1(a).

The transversal symmetry is lifted with the use of the second initial condition, Eq. (6), which possesses a strongly elliptic transverse profile. However, the solution evolves in a way that reduces ellipticity and finally converges to the same radially symmetric optical bullet that was reached with the initial condition of Eq. (5), as shown in Fig. 4. There are decaying oscillations of the elliptical profile of the bullet and corresponding oscillations of the energy before the convergence, but the final state, when the oscillations have died out, is exactly the same. This illustrates the large extension of the basin of attraction, which corresponds to the stationary and stable solution given in Fig. 4(b), for the specific set of parameters written inside the figure.

When the initial condition exits the basin of attraction, the total energy either dissipates or increases to infinity. The latter case is illustrated in Fig. 5. The upper curve in this figure is obtained using the initial condition,

$$
\psi(x, y, t, 0)=4 \exp \left[-\left(\frac{t}{1.3}\right)^{2}-x^{2}-\left(\frac{y}{0.9}\right)^{2}\right]
$$

while the lower one with the use of

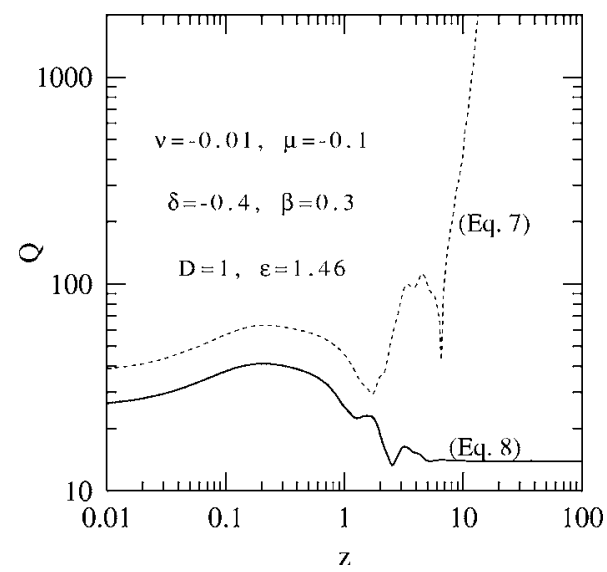

FIG. 5. Energy vs $z$ curves for two different initial conditions obtained with the same set of parameters for the CGLE. The solution with lower energy converges to a stationary dissipative light bullet, whereas the solution with higher energy increases in size, and the value of $Q$ diverges to infinity.

$$
\psi(x, y, t, 0)=4 \exp \left[-t^{2}-x^{2}-\left(\frac{y}{0.8}\right)^{2}\right] .
$$

As explained in Fig. 4(a), an asymmetric initial condition can evolve towards a symmetric stable stationary solution, if it belongs to its basin of attraction. This happens for the evolution of the field initially set by Eq. (8). However, when the initial condition set by Eq. (7) exceeds significantly the limits of the basin of attraction, the subsequent field evolution is characterized by a rapid extension, mostly in the transverse spatial directions, causing the divergence of the total energy. The growth of the transverse size of the field is often accompanied with the formation of intricate patterns. This point is illustrated in the following section.

\section{TYPICAL CASES OF NONSTATIONARY EVOLUTION}

\section{A. Transverse pattern formation in a medium with anomalous dispersion}

In the anomalous dispersion regime, nonstationary patterns expand mainly in the transverse spatial coordinates, thus increasing the total energy. Intricate patterns can be formed in two ways. For the set of parameters admitting stationary bullets, the increase of the energy of an asymmetric initial condition results in its departure from the basin of attraction. The second way is to move and transform the basin of attraction by changing one parameter. For example, we can increase the nonlinear gain.

An example of transverse pattern formation is given in Fig. 6. In this simulation, the nonlinear gain is above the region where stationary bullets exists. A pulse with initially elliptic shape (a) is transformed into a pulse which is elongated in the orthogonal direction $(b, c)$. It splits into several strips and the pattern evolves continuously into a wider structure, never converging to a stationary solution $(d-i)$. The size of the localization increases, both in the transverse direction and in the $t$ domain. It should be recalled that as soon as the field reaches the boundaries $(h, i)$ of the numerical grid, the evolution involves interaction with ghost neighbors, 

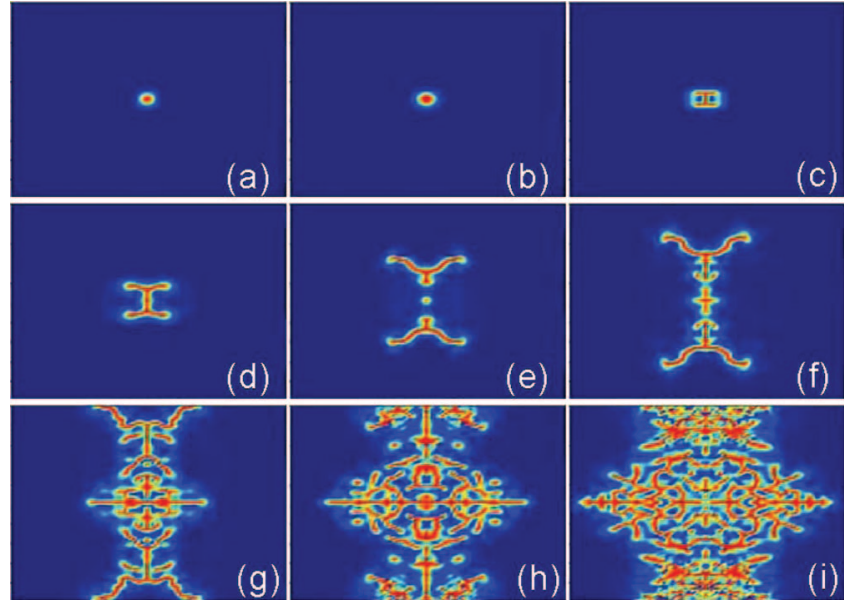

FIG. 6. (Color) Transverse field evolution in propagation when $z$ increases from (a) to (i). The parameters used in the simulation are $\nu=-0.01$, $\mu=-0.1, \delta=-0.4, \beta=0.3, D=1, \epsilon=1.6$. At this set of parameters we observe intricate pattern formation.

due to the periodicity implied by the Fourier transformations used in the computations. Subsequent field evolution with periodic boundary conditions could lead to the formation of regular patterns too, ${ }^{30}$ but we did not investigate this issue.

For higher values of nonlinear gain, the energy growth rate increases significantly. This results in a quick filling in of the transverse space with no intricate patterns being formed. Naturally, the divergence of energy is possible under the assumption of only local saturation effect, as modeled by Eq. (1), and in practice this behavior would be limited at some point by the amount of energy available in the system and global saturation.

\section{B. Rockets in a medium with normal dispersion}

In the case of normal dispersion, the temporal confinement of a light pulse is mainly governed by the nonlinear dissipation. In the absence of dissipation, dispersive linear and nonlinear effects both contribute to the expansion of the field profile with propagation. For a light bullet propagating in a medium with normal dispersion, the temporal direction is more vulnerable to the loss of confinement than the transverse directions. In $x$ and $y$ directions, diffraction and Kerr effects are still partially balanced. The asymmetry of coordinates and the weakness of the soliton in temporal domain are illustrated in the simulations presented in Fig. 7. The transverse profile does not change in this simulation until $z<25$, whereas the temporal profile expands continuously with propagation. As the pulse elongates in the time domain, but remains localized and stationary in the spatial domain, we have dubbed such a solution as "optical rocket."

The elongation process does not last forever. Figure 7(c) shows the evolution of the energy $Q$ versus $z$, for the values of the parameters written inside the figure. From $z=0$ to $z$ $=25$, the energy grows linearly with $z$, due to the linear elongation of the optical bullet as seen in Fig. 7(a). However, this evolution is also unstable. At $z=25$, another instability develops, which destroys the rocket. The rocket "explodes" at both ends, causing a rapid increase of the energy $Q$.
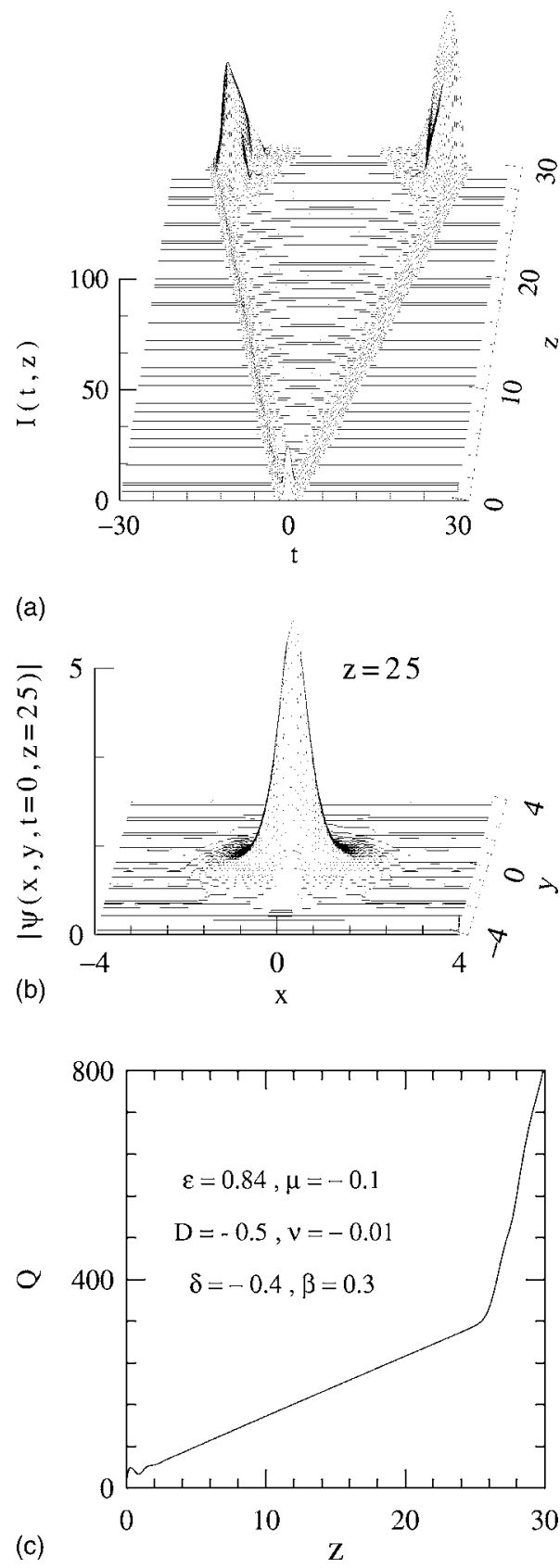

FIG. 7. Pulse evolution leading to the formation of an unstable rocket. (a) The temporal intensity profile, $I(t, z)=\int_{-\infty}^{\infty}|\psi(x, y, t, z)|^{2} d x d y$, elongates in the time domain, while (b) the confinement in transverse directions is maintained. (c) The energy $Q(z)$ in this simulation increases and diverges to infinity. This divergence is accelerated when an "explosion" happens at $z$ $\approx 25$, which is clearly seen in (a). The values of the equation parameters are written in (c).

\section{INTERACTION OF TWO “OPTICAL BULLETS”}

\section{A. Various scenarios of bullet collisions: Nonbinding cases}

The study of the interaction between optical bullets in a three-dimensional space was initiated in Ref. 17, where it was addressed the case of a spherically symmetric CGLE, which implies propagation in anomalous dispersive medium. The interaction problem is complicated, since the outcome of a soliton interaction depends on the initial conditions as well as on the equation parameters. Even though each of the op- 

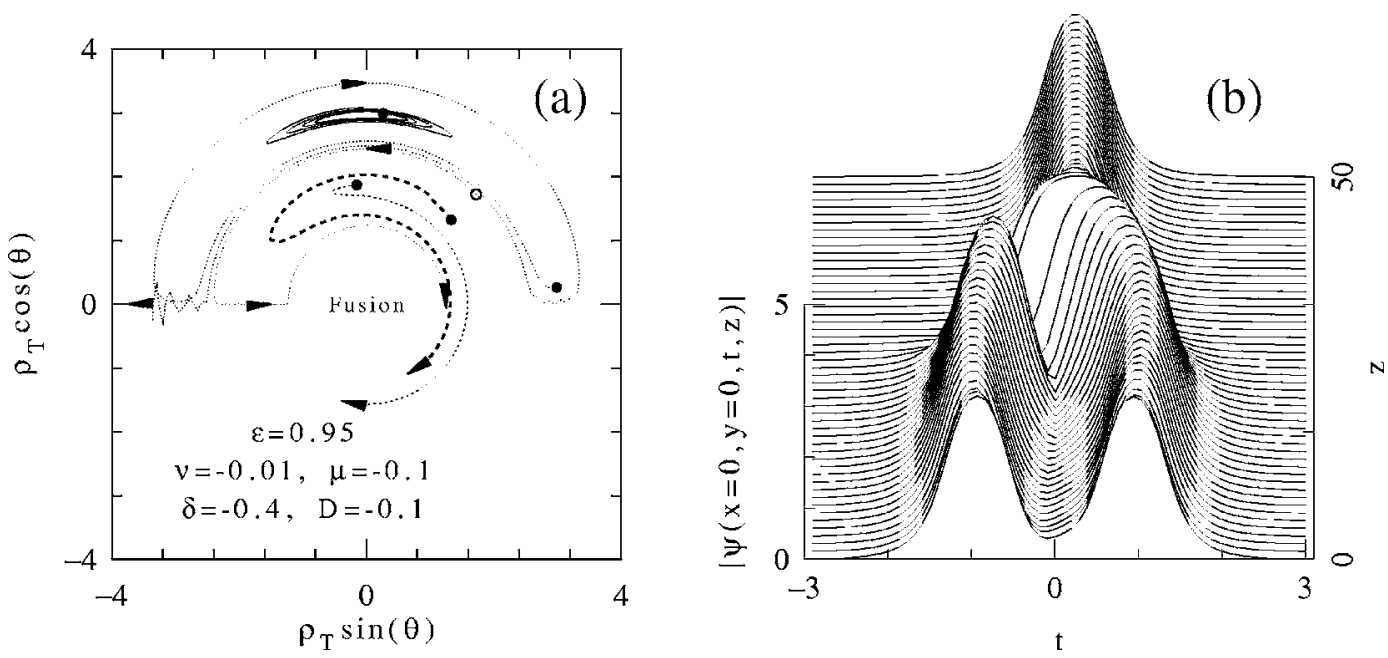

FIG. 8. Interaction of two stable bullets, separated in the temporal domain. Initial relative velocity is zero. (a) Trajectories in the temporal interaction plane. (b) Temporal soliton profiles in the case when the two bullets merge into a single one [thicker dashed line in (a)].

tical bullets has a fixed shape and amplitude, several variables are needed to describe the initial positions and velocities of the solitons. The initial value problem can be stated in terms of the impact parameter and the orientation of the line joining the two bullets in the three-dimensional space. Unlike one-dimensional problems, where only the separation and the phase difference between the solitons matter, ${ }^{28}$ now we have two more angles in the problem. Thus, it is very difficult to perform a complete study of the interaction. Instead, we give only a few examples demonstrating a variety of possibilities. For each case, only stable optical bullets are used in the initial conditions. In the absence of a collision, they would propagate without change indefinitely in $z$.

For illustrative purposes, we show the result of interactions only when the two bullets are initially separated either in time or in space. Further, we choose two different sets of equation parameters corresponding to the normal dispersion regime in one case, and to the anomalous dispersion regime in the other. As in previous sections, we include a spectral filtering term in the temporal domain, so that the spherical symmetry of the CGLE is broken, and only cylindrical symmetry of the CGLE remains, with respect to transverse $(x, y)$ variables.

Clearly, for optical bullets, the interaction plane is not the complete phase space representing the problem. We need more independent variables to show the trajectories uniquely. Although using an interaction plane represents only a subspace of the full phase space of the problem, it is still a helpful tool in this study. Namely, it allows us to illustrate the main outcomes of the interaction. Certain modifications are needed because the two bullets can be separated either in time or in space. Thus, we introduce here temporal or spatial interaction planes, accordingly. We recall that the interaction plane $^{28}$ is a polar set of coordinates where the radius vector corresponds to the separation between the maxima of the two solitons and the angle represents the phase difference between them.

\section{Interaction of two bullets separated in time: Fusion of two bullets}

The initial condition used in the case when the two bullets are separated in time has the form,

$$
\psi(x, y, t, z=0)=\psi_{0}\left(x, y, t-\frac{\rho_{T}}{2}\right)+\psi_{0}\left(x, y, t+\frac{\rho_{T}}{2}\right) \times e^{i \theta},
$$

where $\rho_{T}$ is the initial temporal separation between the bullets and $\theta$ is the phase difference between them. We assume that in spatial transverse plane, each bullet is located at the origin.

Several trajectories in the temporal interaction plane calculated with different initial separations and phase differences are shown in Fig. 8(a). The parameters of the CGLE are written inside the figure. The black dots represent the initial conditions and the arrows show the directions of the trajectories. The final part of each trajectory indicates the outcome of the collision. This figure shows the existence of an unstable focus at $\theta=\pi / 2$, from which trajectories spiral out. A saddle point at $\theta=\pi$ is also clearly seen. On the other hand, when the initial separation is small, the two bullets merge and the trajectories converge towards the origin of the interaction plane. An example of two bullets merging (soliton fusion) is shown in Fig. 8(b).

\section{Interaction of two bullets separated in space}

The initial condition in this case is

$$
\psi(x, y, t, z=0)=\psi_{0}\left(x-\frac{\rho_{R}}{2}, y, t\right)+\psi_{0}\left(x+\frac{\rho_{R}}{2}, y, t\right) \times e^{i \theta},
$$

where $\rho_{R}$ is the initial spatial separation between the two bullets. Separation in time in this case is zero.

The trajectories in the spatial interaction plane displayed in Fig. 9(a) reveal two possible outcomes of the interaction. The trajectories that move away from the origin (mostly at 

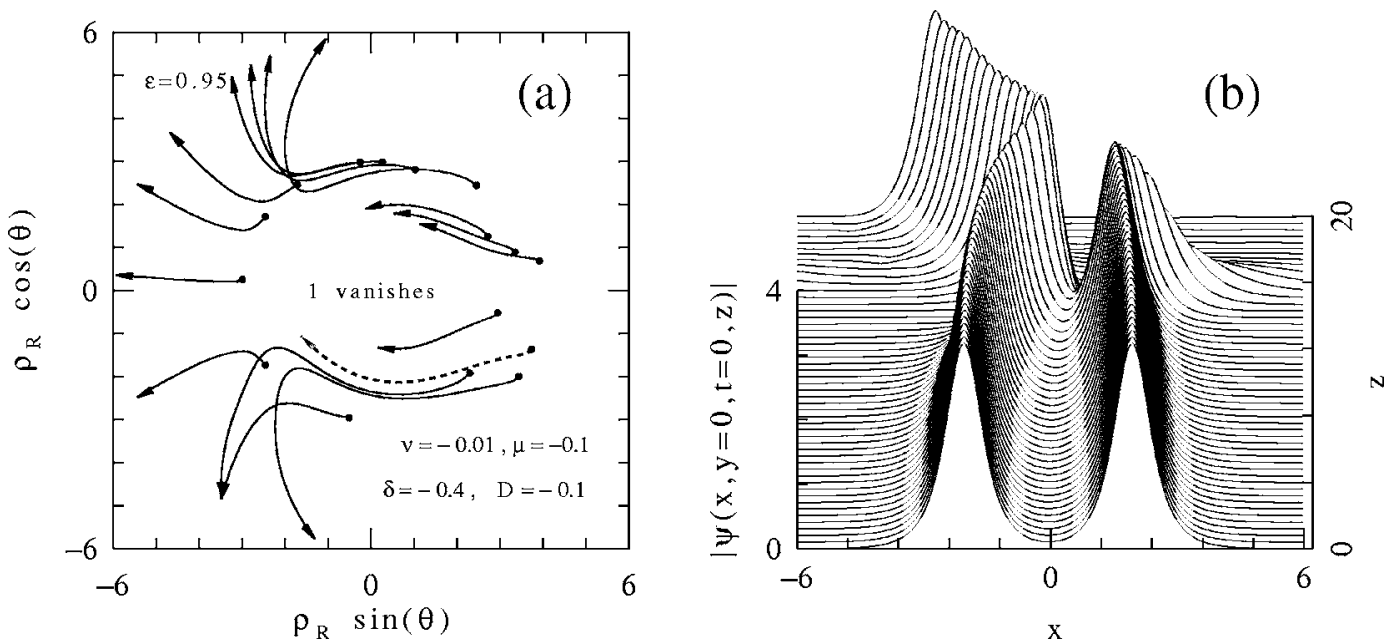

FIG. 9. Interaction of two stable bullets, separated in the spatial domain with zero initial relative velocity. (a) Trajectories in the spatial interaction plane. (b) Soliton profiles in the case when one of the two bullets vanishes.

the left-hand side of the interaction plane) indicate that the separation of the two bullets increases indefinitely. The second type of trajectories move closer to the origin right from the beginning of simulations. In these simulations one of the bullets vanishes. The case of a vanishing bullet is shown in Fig. 9(b). In contrast to the fusion of the two bullets presented in Fig. 8(b), there is no strong overlap between the bullets. In addition, the evolution of the bullets here is strongly asymmetric. The collision results in the recoil upon one bullet and the destruction of the other one. This asymmetry originates from the asymmetric initial phase difference.

\section{Collision of two bullets separated in space}

In the above simulations, we used two bullets with zero velocities in the initial conditions. In contrast to $(1+1)$-D dissipative solitons, optical bullets can have variable velocity in space. This occurs because spectral filtering in our model acts only in the time domain. The velocity is determined by the spatial "chirp" in the initial conditions. Two optical bullets having opposite velocities can collide, provided that they are launched simultaneously in time. Thus, to model a collision of the two bullets moving at a finite angle to each other, we used the following initial condition: $\psi(x, y, t, z=0)=\psi_{0}\left(\mathbf{r}-\mathbf{r}_{A}, t\right) e^{i \mathbf{k}_{A} \mathbf{r}}+\psi_{0}\left(\mathbf{r}-\mathbf{r}_{B}, t\right) e^{i \mathbf{k}_{B} \mathbf{r}+i \theta}$,

where $\mathbf{k}=\left(k_{x}, k_{y}\right)$ and $\mathbf{r}=(x, y)$. In the following, we shall take $\mathbf{k}_{A}=-\mathbf{k}_{B}=-\mathbf{k}$, and then without loss of generality we can choose $\mathbf{k}=(k, 0) . k$ represents then the amount of spatial chirp related to the angle of collision. Our master equation has a symmetry relative to the transformation $\psi_{0}(x, y, t)$ $\rightarrow \psi_{0}(x, y, t) \times e^{i \mathbf{k} r}$. Consequently, if $\psi_{0}(x, y, t)$ is a localized solution of Eq. (1), then $\psi_{0}(x, y, t) \times e^{i \mathbf{k r} r}$ is also a localized solution of this equation, which moves with a velocity proportional to $\mathbf{k}$ in the $(x, y)$ plane.

Once the initial spatial separation and all other parameters of the two bullets are fixed, it is only the phase difference $\theta$ which determines the outcome of the collision. Figure 10 presents two plots of collisions obtained for (a) $\theta=70^{\circ}$ and (b) $\theta=190^{\circ}$. In each example, $k=1$ and $\mathbf{r}_{A}=-\mathbf{r}_{B}=(3,0)$. The soliton profiles in the $x$ direction show the behavior that we can call "repulsion." In case (a) one soliton vanishes after the collision, while in case (b) the two solitons stay intact. Velocities of the two solitons in (b) look the same as before the collision giving to the plot an illusion of an "elastic-type" collision. We have to remember though that the system under consideration is strongly dissipative.

The plots in Fig. 10 provide only the evolution of the field in the $x$ direction when two other variables are fixed $y$
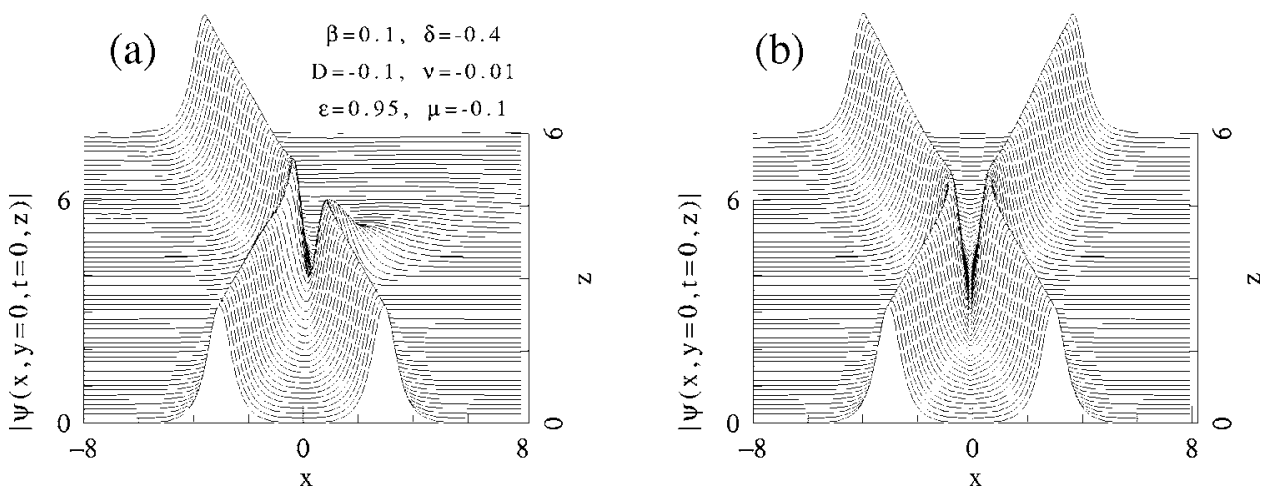

FIG. 10. Collision of two stable bullets initially separated in space and moving towards each other with finite velocities. These examples make an impression of repulsive interaction between the bullets. (a) $\theta=70^{\circ}$. One of the bullets is destroyed by the collision. (b) $\theta=190^{\circ}$. Each bullet survives the collision. Velocities after the collision are roughly the same as before the collision. 

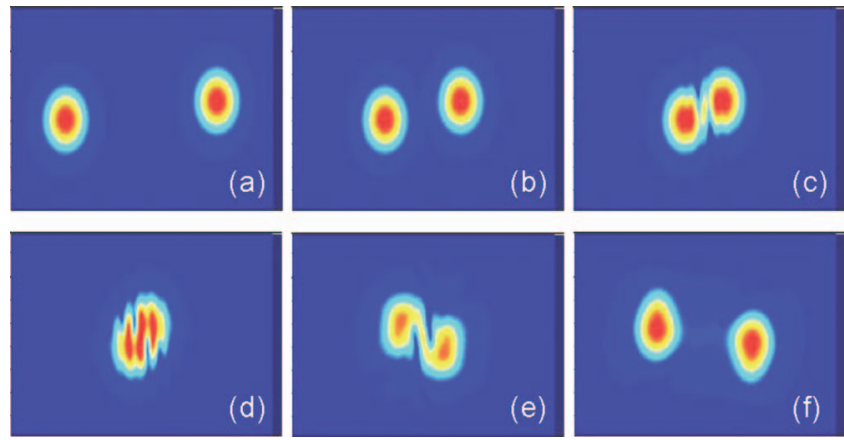

FIG. 11. (Color) (a)-(f): Sequence of a "quasielastic" collision between two stable bullets, separated in the spatial domain with initial relative velocity. The pictures represent the transverse field profiles, and the propagation is in the anomalous dispersion regime.

$=0 ; t=0$. Thus, they give an incomplete description of the collision process. In the theory of particle collisions, there is the impact parameter, $d$, which can be defined as the shortest distance between the two particles if they would move without an interaction. This can be written in terms of the total angular momentum $L$ and the momentum $M$ for the single bullet, i.e., $d=L /(2 M)$, where

$$
L=i \iiint_{-\infty}^{\infty}\left[x\left(\psi_{y} \psi^{*}-\psi_{y}^{*} \psi\right)-y\left(\psi_{x} \psi^{*}-\psi_{x}^{*} \psi\right)\right] d x d y d t
$$

and $M=\sqrt{M_{x}^{2}+M_{y}^{2}}$. The two components of the momentum $M$ are

$$
\begin{aligned}
& M_{x}=i \iiint_{-\infty}^{\infty}\left(\psi_{x} \psi^{*}-\psi_{x}^{*} \psi\right) d x d y d t, \\
& M_{y}=i \iiint_{-\infty}^{\infty}\left(\psi_{y} \psi^{*}-\psi_{y}^{*} \psi\right) d x d y d t .
\end{aligned}
$$

Integration in the two latter equations is assumed across a single bullet. This can be done when the bullets are well separated. Using this definition, the collisions shown in Fig. 10 have a zero-valued impact parameter. Clearly, collisions with a nonzero impact parameter cannot be properly studied with a one-dimensional plot.

A better representation of the collision process can be achieved using a time sequence of $2 \mathrm{D}$ contour plots for the field intensity. We used this representation in Fig. 6 to illustrate pattern formation. This technique gives a much clearer monitoring of soliton collisions, especially when the impact parameter is not zero. For these collisions, the initial angular momentum, $L$, for the system composed of the two bullets is nonzero. For a dissipative system we cannot expect conservation of the angular momentum $L$. However, an initial condition with nonzero angular momentum can trigger a stationary solution with nonzero angular momentum too. Examples can be found in the following section.

Figure 11, plotted using the above technique, illustrates the case of a "quasielastic" collision in the anomalous dispersion regime. The following parameters are used: $D=1$, $\varepsilon=0.66, \delta=-0.4, \beta=0.1, \mu=\nu=-0.1$. The initial condition is given by Eq. (11) with $\mathbf{k}=(3,0), \mathbf{r}_{A}=-\mathbf{r}_{B}=(6,0.5), \theta=0$, yielding an impact parameter of 1 in our dimensionless units. The 2D plot shows that the overlap of the two bullets produces spatial fringes of interference. There is also a small redistribution of energy at the impact region of the interaction, which slightly affects the spatial shape of each bullet after the collision. This collision makes an impression of being quasielastic.

\section{B. Formation of a "bullet molecule": A bound state of two light bullets}

In the $(1+1)-\mathrm{D}$ case, it has been established theoretically and confirmed experimentally that two dissipative temporal solitons can be bound together into a soliton pair. These results were obtained initially for the anomalous dispersion regime and later for the case of the normal dispersion regime (see Chap. 9 in Ref. 2 for an extensive discussion). It was also found that soliton pairs are much easier to find in the anomalous dispersion regime. In order to extend these results to the $(3+1)$-D case, it seems natural to start investigations for the media with anomalous dispersion.

Double bullet complexes do indeed exist for limited regions of equation parameters when correct initial conditions are used. Figure 12 displays an evolution sequence in which such a double bullet complex, or bullet "molecule," is formed as the result of a collision of two well-separated bullets. The following parameters are used in these simulations: $D=1, \varepsilon=0.66, \delta=-0.4, \beta=0.1, \mu=\nu=-0.1$. The initial condition is given by Eq. (11) with $\mathbf{r}_{A}=-\mathbf{r}_{B}=(6,0.25), \mathbf{k}$
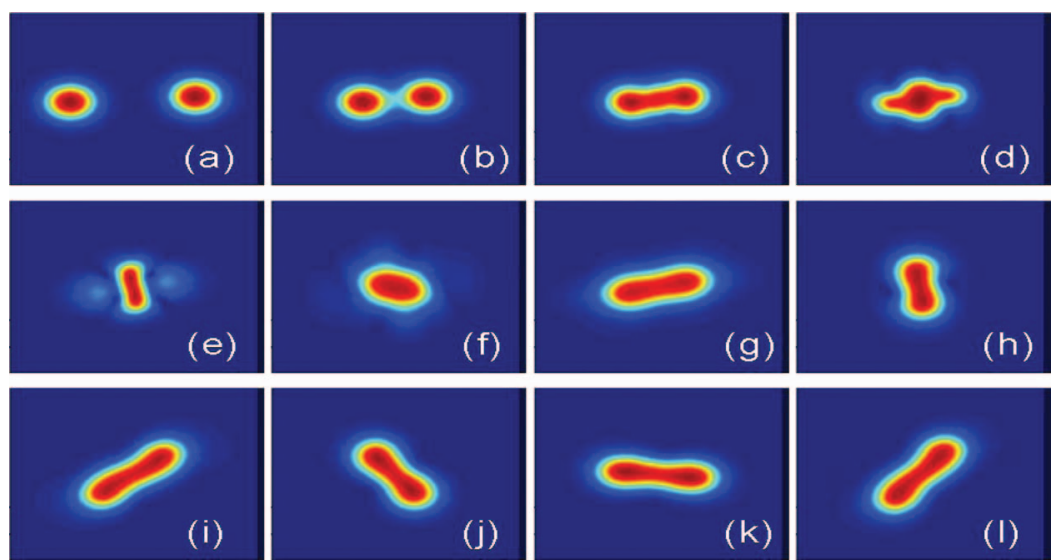

FIG. 12. (Color) Formation of a "bullet molecule," after the collision of two light bullets in the anomalous dispersion regime. $z$ is increasing from (a) to (1). 

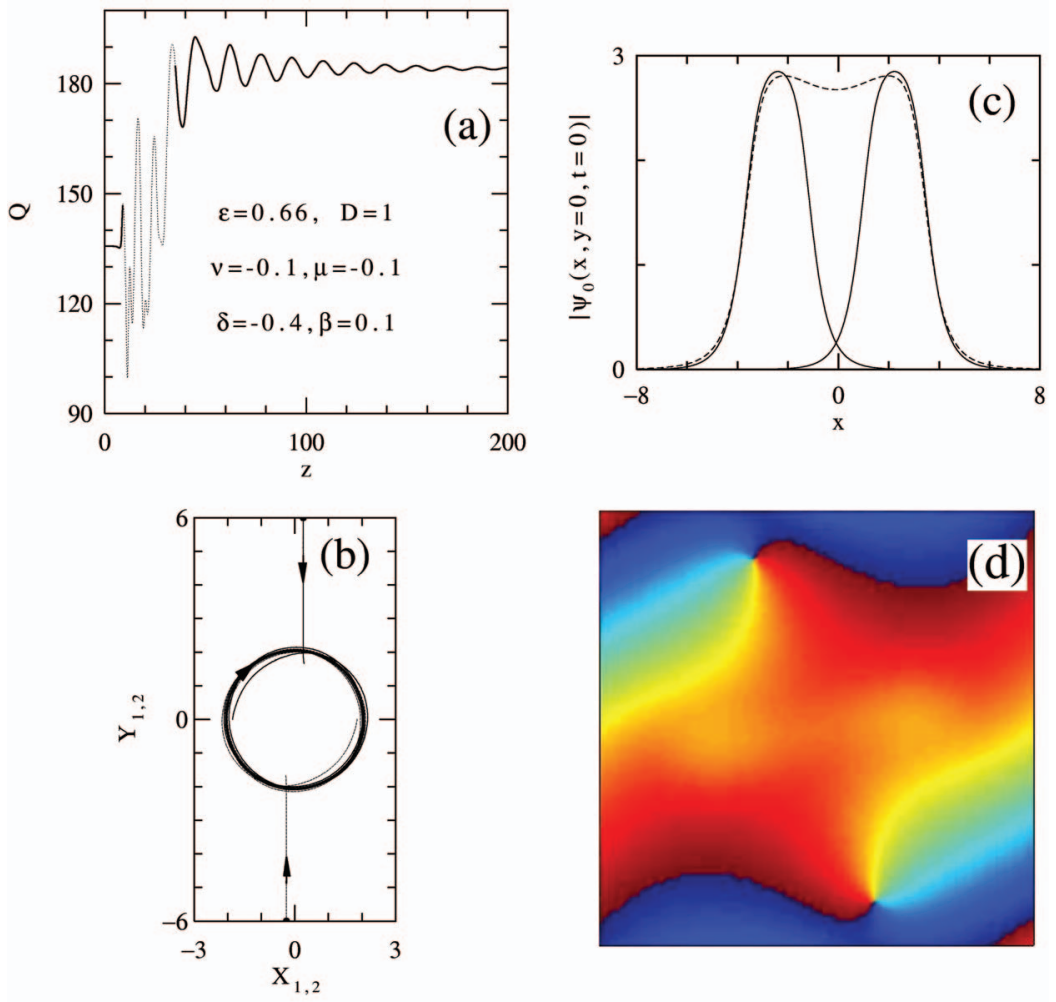

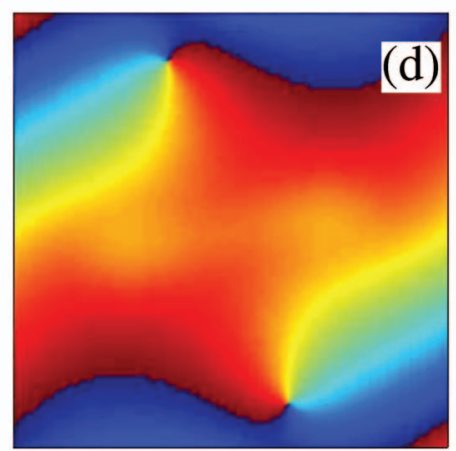

FIG. 13. (Color) Numerical results for a purely rotating double bullet complex. (a) Energy evolution in $z$. During the formation of the complex, the total energy converges to a constant. After the convergence, the complex rotates at constant angular speed around a fixed point in the $(x, y)$ plane. (b) Trajectories of the field maxima in the $(x, y)$ plane for $z$ values in the intervals $[0,9]$ and $[35,200]$ [solid line in (a)]. In the first interval of values of $z$, the respective trajectories are almost two parallel straight lines, while in the second interval, each trajectory converges to a perfect circle (the same for both trajectories). (c) Profiles of two separate bullets (solid lines) and a double bullet complex (dashed line). (d) Color contour plot of the transverse phase profile. The asymmetry of the latter plot reveals the origin of the rotation. In all cases, the values of the equation parameters are those written inside part (a).
$=(0.5,0)$, and $\theta=0$, giving an impact parameter of 0.5 . With respect to the case presented in Fig. 11, the impact parameter is reduced as well as the angle of collision. The latter defines the relative speed at which the collision takes place. As we have found, these are more propitious conditions for binding two light bullets into a complex.

After the complex is formed, the molecule rotates in $2 \mathrm{D}$ space with constant angular velocity. Its shape remains constant in the frame rotating with the complex. The possibility of rotation follows from the three dimensionality of the problem. Nonzero momentum of the initial conditions serves as a seed for the rotation at least at the birth of the rotating molecule. Thus, a nonzero impact parameter is an adequate condition to form a rotating double bullet complex.

\section{ROTATING AND PULSATING DOUBLE BULLET COMPLEXES}

The sequence displayed in Fig. 12 illustrates the formation of a purely rotating double bullet complex, whose dynamics is studied hereafter in Sec. VI A. However, the simple rotation is not the general case of the dynamics of double bullet complexes. The existence of more elaborate double bullet complexes, with energy pulsations, is illustrated in Sec. VI C.

\section{A. Purely rotating double bullet complexes}

The complicated transitional evolution of the dissipative structure at the first stage of the collision in Figs. 12(a)-12(h) is accompanied with large fluctuations and pseudooscillations of the total energy $Q$. Figure 13(a) shows this evolution of the total energy, which finally converges to a constant, when the motion of the double bullet complex be- comes purely rotational. The final value of the energy (around 184) is more than twice the energy of a single bullet (around 68). Thus, the binding energy for the complex is positive and large. The two bullets forming the rotating complex of Figs. 12(i)-12(1) are tightly bound together. The bound state of two bullets can be considered as an optical molecule, since it possesses long-term stability. Moreover, the evolution process described above is analogous to the synthesis of a diatomic molecule out of its two atomic constituents.

The spatial trajectories followed by the two maxima of the field amplitude are provided in Fig. 13(b). These trajectories are not plotted for the whole evolution depicted in Fig. 12 , as during the climax of the collision one cannot distinguish two entities. The trajectories are shown for the $z$-evolution part plotted as a solid line in Fig. 13(a). Figure 13(b) clearly shows the final circular trajectories for each bullet in the bound state. Figure 13(c) shows the profile $\left|\psi_{o}(x, y=0, t=0)\right|$ of two independent single optical bullets (solid lines), conveniently displaced, together with the profile of the rotating double bullet complex (dashed line). They represent the profiles of each colliding bullet and the resulting complex after the collision in Figs. 13(a) and 13(b). Both formations are stable simultaneously for this specific set of equation parameters, which is the same as in the whole figure [written inside part (a)]. This figure validates the idea that the complex is indeed a composite state of two stationary optical bullets. The match of the temporal profiles for these two types of solutions (not shown) is almost perfect. The distance between the two bullets in the complex is comparable to the width of each soliton. Consequently, the strength of the bond between the two bullets is comparable to the energy of each bullet. 
The double bullet complex can be excited from a variety of initial conditions. Two stationary bullets located far apart from each other but still interacting through their tails can form a complex. If the relative phase difference between the bullets is chosen properly, we observe an attraction and transformation of this solution into a bound state. The resulting complex appears to have a fixed angular velocity but the rotation could occur in either direction (clockwise or counterclockwise), depending on the sign of the initial angular momentum. In the absence of initial angular momentum, its role is played by unavoidable numerical noise. In the stationary regime, the reason for rotation can be seen in the rotational asymmetry of the phase contour plot in the plane $(x, y)$ at $t=0$. The latter is illustrated in Fig. 13(d). This relationship between the phase distribution and the rotational motion of a nonlinear optical structure has been studied in the general case by Rosanov. ${ }^{31}$

\section{B. Regions of existence of double bullet complexes}

Stable single bullets as well as stable double bullet complexes do exist in both chromatic dispersion regimes. ${ }^{32}$ The regions of existence of the complexes in the space of the system parameters can be obtained the same way as those of stable single bullets. To find these regions, we restricted ourselves to varying only two parameters as we did before with single bullets, This time we chose $\nu$ and $\varepsilon$ as variable parameters, while fixing the four others. This is in contrast to varying $D / \beta$ and $\varepsilon$ as in Fig. 3. The reason for this change is that the small value of the nonlinear index saturation $\nu=-0.01$ used in Fig. 3 did not result in the appearance of stable bullet complexes. Keeping the dispersion as a constant and varying the nonlinear index saturation allowed us to unveil the formation of various double bullet complexes. We have found a wide range of complexes from stable, purely rotating states presented in Sec. VI A, to strongly pulsating complexes that are described below in Sec. VI C. Figure 14 summarizes our results in obtaining regions of existence of stable double bullet complexes. As expected, the region of existence of the complexes is smaller than that for single bullets.

We studied normal and anomalous dispersion regimes separately. Figures 14(a) and 14(b) illustrate these two situations. The hatched areas in each plot show 2D sections of the region of existence of stable single optical bullets in the $6 \mathrm{D}$ parameter space. The smaller shaded regions are those regions that admit stable double bullet complexes. Although the regions of existence may seem quite similar in the two dispersion regimes, there is a significant difference in terms of coexistence between the single bullets and double bullet complexes. Indeed, for the anomalous dispersion case, the region of existence of stable double bullet complexes resides completely inside the region of existence of stationary single bullets. This is hardly surprising because, to have a stable composite state of two bullets, one would expect that each of them should be stable. However, the two regions may overlap only for the set of the parameters that we have chosen: changing some of the other four parameters of the equation may separate them. This is actually the situation met in Fig. 14(b). The region for double bullet complexes has a part
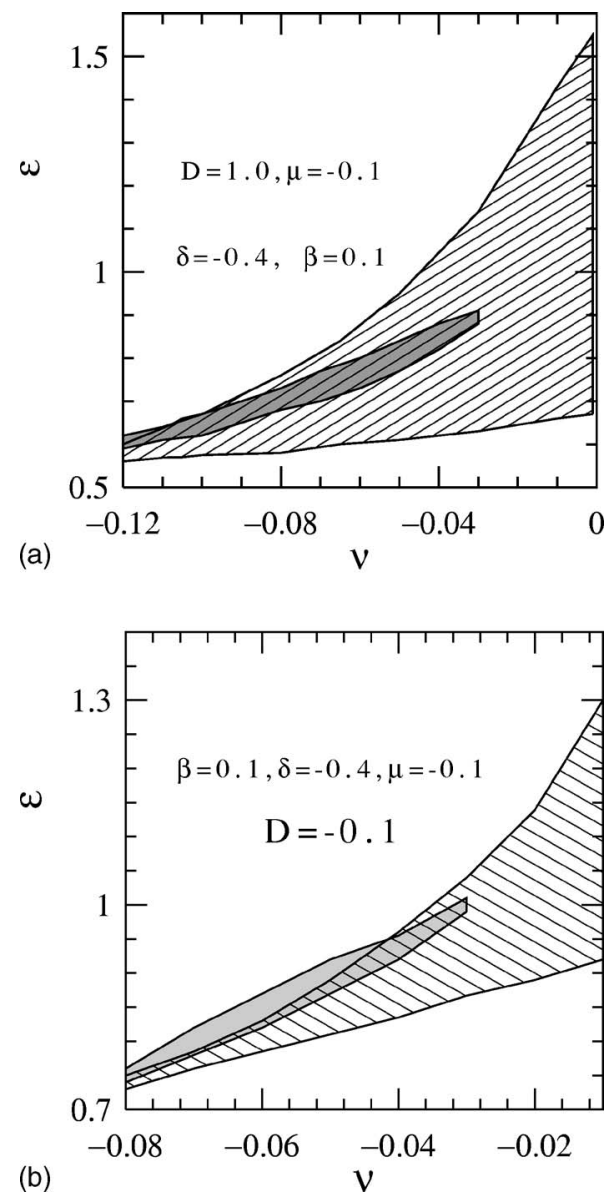

FIG. 14. Regions of existence in the plane of parameters $(\epsilon-\nu)$ for single bullets (hatched area) and stable double bullet complexes (in gray), in the case of (a) anomalous and (b) normal dispersion regimes.

located outside of the region for stationary single bullets. Thus, for the normal dispersion case, there is a region where only double bullet complexes exist in addition to a common region of simultaneous existence of the two types of localized solutions.

The fact that double bullet complexes can exist in the region where there is no stable single bullet can be understood if we recall the large binding energy of double bullet complexes. A double bullet complex is not just the sum of two single bullets [see Fig. 13(c)].

\section{Internal motions with one or two oscillation periods}

The existence of pulsating localized solutions is a general feature of nonlinear dissipative systems. In the present case, pulsating solutions can be found close to the boundaries of the regions of existence of stable stationary soliton solutions. A pulsating solution can be attributed to the existence of a limit cycle in the system. Limit cycles can be stable or unstable just like isolated fixed points that correspond to stationary solitons. Stable pulsating dissipative optical solitons were found in the $(1+1)$-D temporal case in Ref. 29. Several types of pulsations were found also for temporal soliton pairs. The most general type of pulsations in the latter case is due to oscillations of the temporal separation 

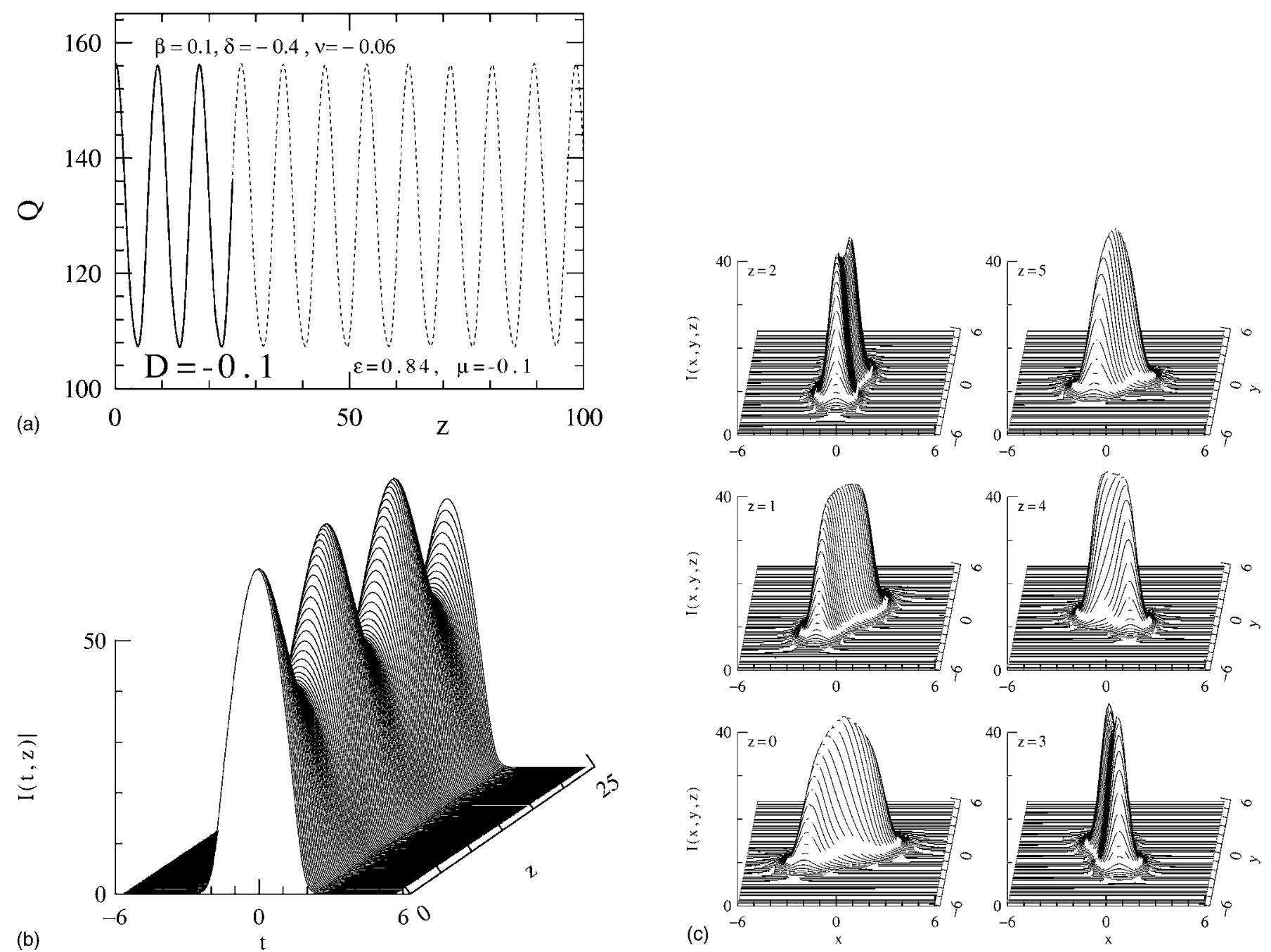

FIG. 15. Numerical simulations illustrating "harmonic rovibration" of a double bullet complex. (a) Evolution of the energy $Q$ of the localized solution along the $z$ axis. The total energy is almost a sinusoidal function of $z$. (b) Periodic evolution of the temporal field profile with $z$ for the same simulation. (c) The transverse field intensity profile as defined by Eq. (12), at several values of $z$ inside a half of a period. A combined motion of rotation and vibration for this solution is clearly seen.

and phase difference between the two bound solitons. These oscillations are very similar to the vibrational motion of a molecule. $^{33,34}$

We extended these results to the (3+1)-D spatiotemporal case, revealing the existence of pulsating optical bullet complexes. ${ }^{32}$ Pulsating double bullet complexes can also be found for both anomalous and normal dispersion cases. Using the terminology accepted in molecular physics, we talk about "rovibrational" motion of soliton complexes. In addition to quasiharmonic rovibrational motions, we found two types of nonharmonic motions, observed in the anomalous dispersion regime; first, the onset of pulsations having two oscillation periods. Secondly, we found period-2 pulsations obtained after two successive bifurcations. Other period- $N$ $(N \geqslant 3)$ pulsations were also observed, but will not be treated here.

\section{Harmonic pulsations: Rovibrational motion}

Pure rotations of a bullet complex are characterized by the constant energy of the localized solution. Any additional internal motion manifests itself in energy pulsations. For molecules, the most general relative motion to imagine comprises the oscillation of the relative distance separating the two bound bullets, namely a vibrational motion. In combination with the rotation described in Sec. VI A, this motion leads to a rovibrating double bullet complex. This type of solution is illustrated in Fig. 15. In particular, Fig. 15(a) shows the evolution of the total energy along the $z$ axis, which is oscillating almost harmonically. The values for the system parameters are written inside the figure. Note that the dispersion in this case is normal. The integrated temporal profile of this solution is shown in Fig. 15(b), which displays oscillations of the profile along $z$ with the same period as the energy. A sequence of spatial profiles at various values of $z$ presented in Fig. 15(c) reveals the combined motions of rotation and vibration. The strength of vibration is such that the full overlap of the two bullets occurs at every oscillation period. Along the vertical axis, we plot the quantity $I(x, y, z)$ defined as the integral of intensity across the time variable, viz., 


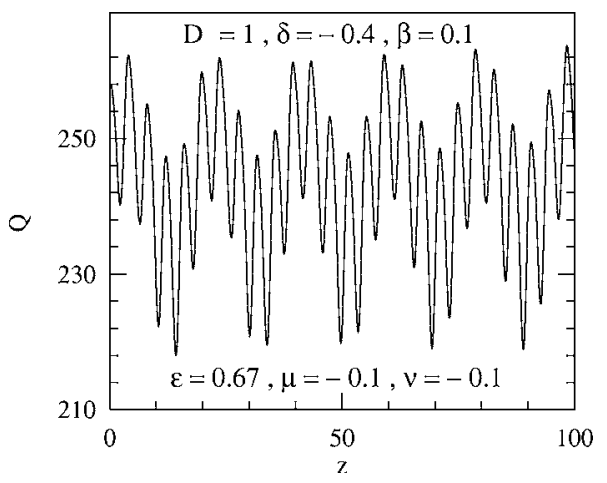

FIG. 16. Energy vs $z$ for a double bullet complex pulsation with two oscillation periods.

$$
I(x, y, z)=\int_{-\infty}^{\infty}|\psi(x, y, t, z)|^{2} d t
$$

For a nonlinear system, harmonic oscillations can be found near a point of Hopf bifurcation from a fixed point to a limit cycle. As a general rule, the spectral content of the energy oscillations tends to increase with the amplitude of these oscillations when parameters move further away from the bifurcation. It is surprising to find, in a highly nonlinear dynamical system, harmonic pulsations associated with relatively large amplitude oscillations of the total energy. In the case presented in Fig. 15, energy pulsations remain very close to harmonic pulsations, even for an amplitude of modulation, which is almost $20 \%$ of the average total energy.

\section{Nonharmonic pulsations}

The variety of oscillating behaviors that can be observed when altering the system parameters is enormous. In particular, we noticed the strong influence of the parameter $\nu$ on self-pulsations. Although the change of the dispersion regime strongly affects the dynamics, we observed harmonic as well as nonharmonic pulsations for each sign of the dispersion. In this paper, nonharmonic pulsations are presented for the anomalous dispersion regime.

To show pulsations with two oscillation periods, the system has to undergo two bifurcations from the stationary solution. When adequate parameter changes are found, the asymptotic evolution of the total energy leads to a pulsation mode whose spectrum contains two main frequencies. Far from the bifurcation points, the two spectral amplitudes can be of the same order of magnitude. A vivid example of such evolution of the total energy for a two-frequency pulsating solution is given in Fig. 16. The thorough analysis of the motion associated with these pulsations is far from being trivial. The details can be found in Ref. 32 .

In addition to previously known results, in this paper, we present a newly observed behavior, namely the onset of period-2 pulsations in double bullet complexes. Period doubling bifurcations are common in nonlinear dynamics of finite dimensional as well as infinite dimensional systems. They have been observed in a variety of physical problems starting from the simplest example: the logistic map. Period doubling bifurcations of pulsating dissipative solitons of the $(1+1)$ D CGLE have been studied in Ref. 35. The compli- cated dynamics that arises in the latter case is related to the multiplicity of external parameters involved in the problem. However, the sequence of period doubling bifurcations leading to the chaotic evolution of the soliton, can be observed even in this case. ${ }^{35}$

Figure 17(a) shows a bifurcation diagram obtained when varying the nonlinear gain parameter $\varepsilon$, while keeping the rest of parameters (written inside the figure) fixed. Each point represents a local maximum or minimum of the $Q(z)$ function obtained for the given set of parameters, once any transitory behavior has been removed. For a large interval of the $\varepsilon$ values, $Q$ is single valued. This means that the energy does not depend on $z$. At $\epsilon=0.868$ a bifurcation occurs, which is related to the onset of pulsations with single frequency. As we increase $\varepsilon$ up to 0.879 , the period doubling bifurcation occurs.

Figure 17(b) shows the asymptotic evolution of $Q$ versus $z$ at $\varepsilon=0.882$ [indicated in (a) by a vertical dashed line], illustrating the period-2 pulsations. The relative motion of the two bullets is illustrated in Figs. 17(c) and 17(d). The first one shows the evolution of the distance, $\rho_{R}$, between the two peak amplitudes, while the second one shows the trajectories of these peaks in the $(x, y)$ plane. The relative separation of the two bullets oscillates in accordance with the oscillation of the total energy. When the two bullets move farther away from each other, the field amplitude between the bullets, i.e., at $x=0$, hardly reduces. This can be seen from Fig. 13(c). The nonlinear gain (dissipation) in the system allows for large variations of the total energy. This is of course a major difference with the Hamiltonian dynamics of matter molecules.

The transverse motion of the double bullet complex is a combination of rotation and vibration just as in the previous case. The two bullets remain perfectly distinguishable during the whole motion. Each oscillation of $Q$ results in one loop of the trajectory in the $(X, Y)$ plane that looks like a distorted circle. The part of the trajectory for each bullet shown for the given distance $z$ consists of two distorted circles.

\section{CONCLUSION}

The reviewing of the results related to dissipative light bullets allows us to draw a few important conclusions. It is clear that most of the fundamental results obtained previously in the realm of $(1+1)$-D dissipative temporal solitons can be generalized to the case of the full $(3+1)$-D spatiotemporal solitons. Among them, we can mention the following: (a) The balance between gain and loss, plus the interplay between dispersion and nonlinearity, allows to form stable bell-shaped solitons, both in the normal and in the anomalous dispersion regimes. (b) Dissipative solitons can be stationary or pulsating. (c) Dissipative solitons can form stable bound double bullet complexes. (d) Double bullet complexes can also be stationary or pulsating. The latter fact is based on the existence of limit cycles in nonlinear dissipative systems. Thus, the notion of dissipative solitons developed earlier for $(1+1)$-D systems with all its fundamental consequences can be eagerly extended to the $(3+1)$-D spatiotemporal case. No 

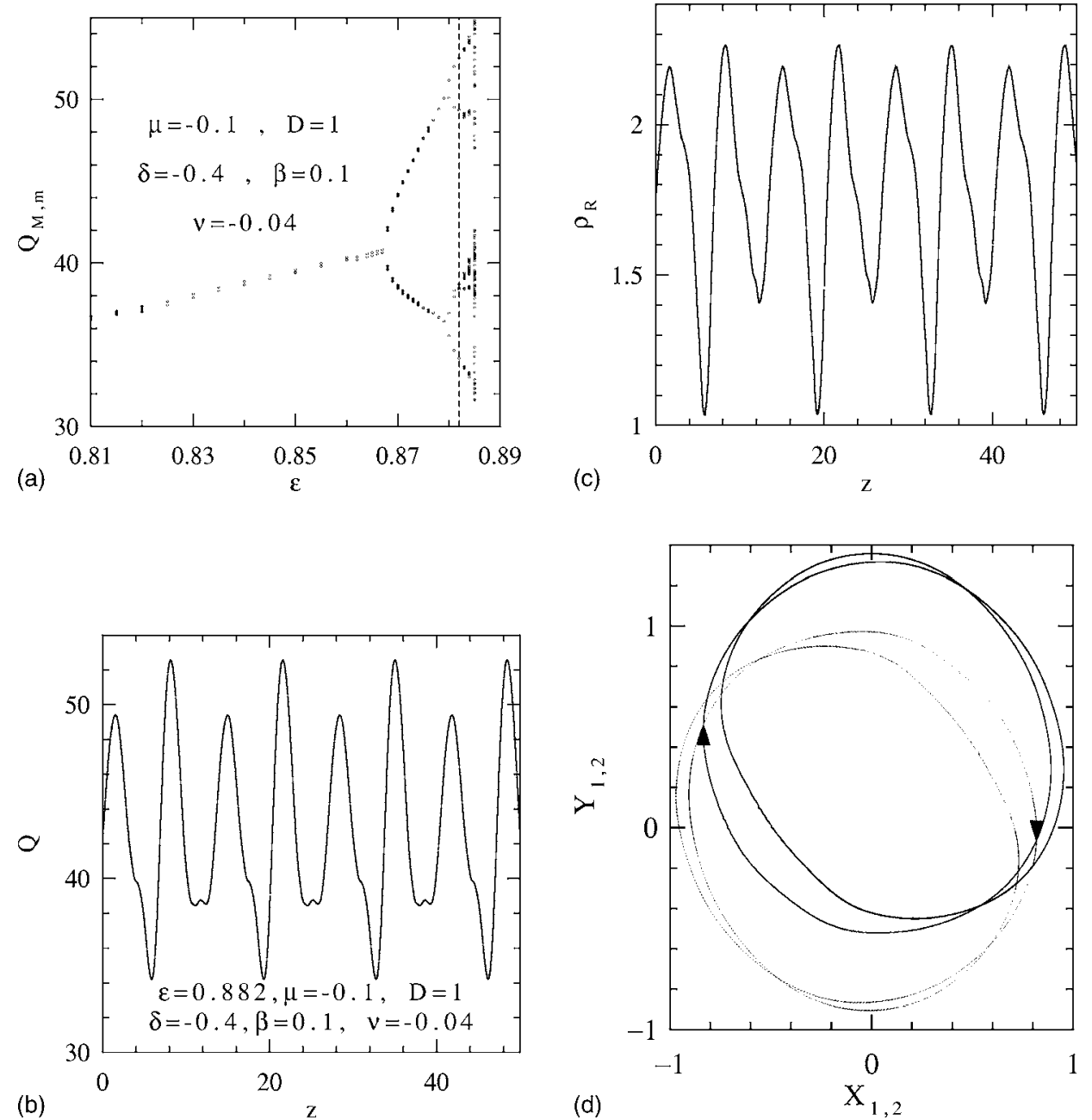

FIG. 17. Bifurcations and period-2 pulsations of a double bullet complex. (a) The local extrema $Q_{M, m}$ of the total energy $Q$ vs $\varepsilon$ (bifurcation diagram). Transition to pulsations (at $\varepsilon=0.867$ ) and period doubling bifurcation (at $\varepsilon$ $=0.879$ ) are clearly seen. (b) Oscillations of energy $Q(z)$ for the period-2 pulsations at $\varepsilon=0.882$. This value is marked by the vertical dashed line in (a). (c) The relative separation of the two bullets $\rho_{R}$ vs $z$. This plot shows nonharmonic vibrational motion of the complex. (d) Spatial trajectories of the two field maxima on the transverse $(x, y)$ plane doubts, dissipative light bullets have additional features that are impossible in $(1+1)$-D systems. An example is rovibrations of double bullet complexes. Thus, studies of $(3+1) \mathrm{D}$ spatiotemporal dissipative solitons are well justified.

However, the $(3+1) \mathrm{D}$ spatiotemporal case stays a very hard problem to tackle in its generality. Numerical simulations are tedious and time consuming even with the latest fast computers. Exploration of the regions of existence for light bullets as well as the unveiling of their complex dynamics require enormous computer power. Only partial investigations were possible, as the CGLE has six independent parameters to vary. In that perspective, complementary studies that use methods derived from a variational approach can be useful in preliminary mapping of parameter domains of interest. ${ }^{36}$ However, only full computations can assert or cancel the dynamics found in such a way. The necessity of using various initial conditions add more difficulties to the problem. This is particularly true when addressing the case of dissipative bullet complexes. The variety of interaction and collision scenarios is so large that it would be impossible to present them all in one publication. Complex rovibrational dynamics undoubtedly deserves more studies. As we already know from the $(1+1)$-D temporal case, when the number of solitons in a bound state exceeds 2 , the investigation becomes much more complicated. Until now, we have not undertaken such a study in the $(3+1)$-D case. We firmly believe that stable complexes formed by three, four, etc., light bullets do exist the same way as temporal multisoliton complexes in $(1+1)$-D systems, with the additional freedom provided by the higher dimensionality. Thus, they can be compared with polyatomic matter molecules.

In this paper, we have presented only a limited number of examples of soliton dynamics, carefully chosen and simulated numerically with great care. Thus, we were able to unveil only a small part of the wonderful world of dissipative solitons and their complexes in $(3+1) \mathrm{D}$. No doubt, the work will be continued and will find relationships and applications in various fields in physics, chemistry, and biology that can be described by the complex Ginzburg-Landau equation. As a particular example, we can mention future massive parallel preprocessing and dynamical routing of optical data. At the present state of the art, it can be a futuristic view, but dissipative solitons surely have many advantages when used as optical bits of information.

\section{ACKNOWLEDGMENTS}

N.A. acknowledges support from the Australian Research Council. The work of J.M.S.-C. was supported by the M.E.y C. under Contract No. FIS2006-03376 and P.G. acknowledges support from Agence Nationale de la Recherche. 
${ }^{1}$ G. Nicolis and I. Prigogine, Self Organization in Nonequilibrium Systems-From Dissipative Structures to Order Through Fluctuations (Wiley, New York, 1977).

${ }^{2}$ Dissipative Solitons, edited by N. Akhmediev and A. Ankiewicz (Springer, Heidelberg, 2005).

${ }^{3}$ Y. Silberberg, Opt. Lett. 15, 1282 (1990).

${ }^{4}$ D. E. Edmundson and R. H. Enns, Phys. Rev. A 51, 2491 (1995).

${ }^{5}$ C. Josserand and S. Rica, Phys. Rev. Lett. 78, 1215 (1997).

${ }^{6}$ N. Akhmediev and J. M. Soto-Crespo, Phys. Rev. A 47, 1358 (1993).

${ }^{7}$ Y.-F. Chen, K. Beckwitt, F. Wise, and B. A. Malomed, Phys. Rev. E 70, 046610 (2004).

${ }^{8}$ H. Meng, G. Salamo, M.-F. Shih, and M. Segev, Opt. Lett. 22, 448 (1997).

${ }^{9}$ D. Skryabin and W. Firth, Opt. Commun. 148, 79 (1998).

${ }^{10}$ X. Liu, L. J. Qian, and F. W. Wise, Phys. Rev. Lett. 82, 4631 (1999).

${ }^{11}$ B. A. Malomed, D. Mihalache, F. Wise, and L. Torner, J. Opt. B: Quantum Semiclassical Opt. 7, R53 (2005).

${ }^{12}$ L. Torner and A. Barthélémy, IEEE J. Quantum Electron. 39, 22 (2003).

${ }^{13}$ L. Jankovic, P. Aboussan, M. Affolter, and G. Stegeman, Opt. Express 12, 5562 (2004).

${ }^{14}$ A. Buryak, P. Di Trapani, D. Skryabin, and S. Trillo, Phys. Rep. 370, 63 (2002).

${ }^{15}$ C. Conti, S. Trillo, P. Di Trapani, G. Valiulis, A. Piskarskas, O. Jedrkiewicz, and J. Trull, Phys. Rev. Lett. 90, 170406 (2003).

${ }^{16}$ P. Di Trapani, G. Valiulis, A. Piskarskas, O. Jedrkiewicz, J. Trull, C. Conti, and S. Trillo, Phys. Rev. Lett. 91, 093904 (2003).

${ }^{17}$ N. N. Rosanov, Spatial Hysteresis and Optical Patterns (Springer, Berlin, 2002), Chap. 6.

${ }^{18}$ S. Barland, J. Tredicce, M. Brambilla, L. Lugiato, S. Balle, M. Giudici, T. Maggipinto, L. Spinelli, G. Tissono, T. Knödl, M. Miller, and R. Jäger, Nature (London) 419, 699 (2002).
${ }^{19}$ E. Ultanir, G. Stegeman, Ch. Lange, and F. Lederer, Opt. Lett. 29, 283 (2004)

${ }^{20}$ M. Brambilla, T. Maggipinto, G. Patera, and L. Columbo, Phys. Rev. Lett. 93, 203901 (2004).

${ }^{21}$ W. Firth and A. Scroggie, Phys. Rev. Lett. 76, 1623 (1996).

${ }^{22}$ J. M. Soto-Crespo, N. N. Akhmediev, and V. V. Afanasjev, J. Opt. Soc. Am. B 13, 1439 (1996).

${ }^{23} \mathrm{Ph}$. Grelu, J. M. Soto-Crespo, and N. Akhmediev, Opt. Express 13, 9352 (2005).

${ }^{24}$ J. M. Soto-Crespo, Ph. Grelu, and N. Akhmediev, Opt. Express 14, 4013 (2006).

${ }^{25}$ L.-C. Crasovan, Y. V. Kartashov, D. Mihalache, L. Torner, Y. S. Kivshar, and V. M. Perez-Garcia, Phys. Rev. E 67, 046610 (2003).

${ }^{26}$ M. Stratmann, T. Pagel, and F. Mitschke, Phys. Rev. Lett. 95, 143902 (2005)

${ }^{27}$ J. M. Soto-Crespo, N. Akhmediev, and K. Chiang, Phys. Lett. A 291, 115 (2001).

${ }^{28}$ N. Akhmediev and A. Ankiewicz, Solitons, Nonlinear Pulses and Beams (Chapman \& Hall, London, 1997).

${ }^{29}$ J. M. Soto-Crespo, N. N. Akhmediev, V. V. Afanasjev, and S. Wabnitz, Phys. Rev. E 55, 4783 (1997).

${ }^{30}$ M. Tlidi and P. Mandel, Phys. Rev. Lett. 83, 4995 (1999).

${ }^{31}$ N. N. Rosanov, Opt. Spectrosc. 96, 405 (2004).

${ }^{32}$ J. M. Soto-Crespo, N. Akhmediev, and Ph. Grelu, Phys. Rev. E 74, 046612 (2006).

${ }^{33}$ M. Grapinet and Ph. Grelu, Opt. Lett. 31, 2115 (2006).

${ }^{34}$ J. M. Soto-Crespo, Ph. Grelu, N. Akhmediev, and N. Devine, Phys. Rev. E 75, 016613 (2007).

${ }^{35}$ J. M. Soto-Crespo, M. Grapinet, Ph. Grelu, and N. Akhmediev, Phys. Rev. E 70, $066612(2004)$

${ }^{36}$ V. Skarka and N. B. Aleksić, Phys. Rev. Lett. 96, 013903 (2006). 